\title{
Modeling GSM Based Network Communication in Vehicular Network
}

\author{
M. Milton Joe \\ Assistant Professor, Department of Computer Application, \\ St. Jerome's College, Nagercoil, Tamilnadu, India. \\ Email: m.miltonjoe@gmail.com \\ Dr. B. Ramakrishnan \\ Associate Professor, Department of Computer Science and Research Centre, \\ S.T. Hindu College, Nagercoil, Tamilnadu, India. \\ Email: ramsthc@gmail.com \\ Dr. R. S. Shaji \\ Professor, Department of IT, Noorul Islam University, Nagercoil, Tamilnadu, India. \\ Email: shajiswaram@yahoo.com,
}

\begin{abstract}
Obviously fair communication establishment in every technology increases the efficiency. As we know well, vehicles are used in day to day life of every human being to move from one location to another location. If network communication is formed between vehicles, mobile phones and home based telephones, it will increase the safety of the passengers by communicating with one another. In this paper, we propose GSM based network communication in vehicles, which will develop reliable network communication between vehicles, mobile phones and home based telephones. The added advantage GSM based network communication among vehicles will lead to safety of travel by tracking the vehicle's location, since GSM based network communication is established in vehicles.
\end{abstract}

Index Terms - VANET, Network Communication, GSM, Security, Location Tracking, Efficiency, Safety.

\section{INTRODUCTION}

Modeling network communication with two or more vehicles, while move on the road is called VANET [1] [2] [3]. This vehicular network is a wireless network communication established with wireless technologies [1]. This vehicular network communication is also similar to mobile ad-hoc network called MANET, where in MANET the nodes could move at random and in VANET the nodes can move only on the prescribed roads [1] [2] [4]. The same protocol used in MANET can be used in VANET also to transmit and receive the messages [1] [2]. All this network communication is established between vehicles only. In this paper, we model a new network communication that establishes the communication among vehicles, mobile phones, help lines and home based telephones.
The above Fig. 1 represents the simple way of communication established in GSM based vehicles. That is network communication is established between GSM based vehicles, mobile phones, help lines and home based telephones

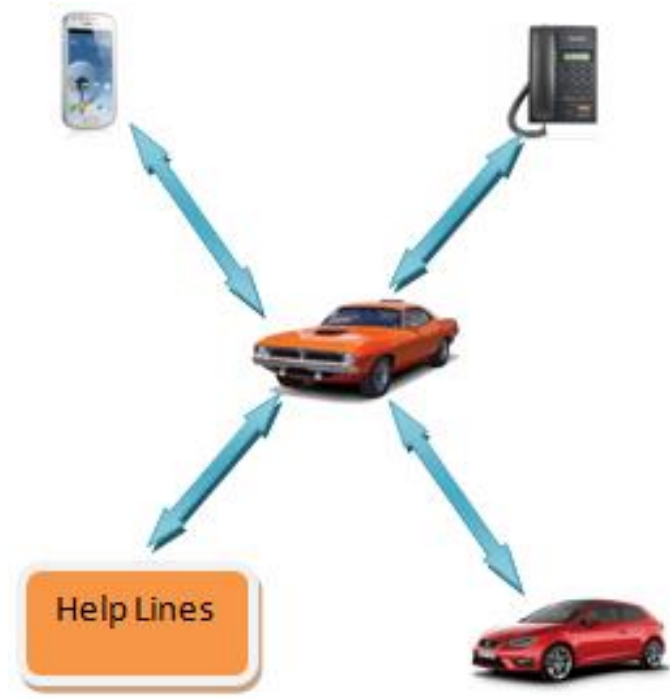

Figure. 1: GSM based Communication

The various types of communication can be such as Short Message Service (SMS), Voice Call, Multimedia Messaging Service (MMS) and vehicle tracking system. This sort of network communication established in vehicles will help a lot to communicate with one another in an easy manner and especially the vehicle can traced out easily, when hijack or some sort of disaster happens to the vehicles, while moving on the road. As all know well vehicle move even very faster on the highway. In this situation unexpected things may occur to the vehicle. At that time the driver or the passengers could be able to communicate with the other sources such as help lines found on the highway 
or even to other mobile devices. In this emergency situation the vehicle should be equipped to communicate with others sources to protect it. In order to bring the safety of the passengers and the vehicle some sort of network communication should be formed. In this paper we have proposed and implemented GSM based network communication in the vehicle to form the network communication among the vehicle, mobile phones, and help lines on highway and home based telephones. The proposed scheme increases the safety of the passengers along with the vehicle during the emergency cases. This type of GSM based communication is ultimately needed to track the entire history of the vehicle, when some serious mishap or hijack of the vehicle takes place.

The proposed model establishes network communication among vehicles, mobile phones, help lines and home based telephone. Once communication is established among them, they can communicate with one another with the help of GSM technology. GSM technology should be included in the vehicle during manufacturing it. This GSM communication model allows to exchange messages with other vehicle, mobile phone, help lines and home based telephone. GSM communication model not only used for communication but also it is used to track the entire history of the vehicle, wherever it has traveled. This vehicle tracking model helps a lot during hijack. The rest of the paper represents.

\section{ATED WORK}

Vehicular communication is almost similar to the MANET communication. In MANET communication the nodes can be moved from one location to another location at random fashion, however in VANET the nodes are considered as vehicles that could move only the road [5]. VANET is the most advanced version of MANET and all the features of MANET could be applied to VANET also [5]. VANET enables the communication between moving vehicles and the Road Side Units (RSU) that are available on the highways and the city environment [6]. The On Board Unit (OBU) is equipped to transmit and receive the messages between vehicle and RSU [7]. To provide safer travel Intelligent Transport System (ITS) was developed and it provides necessary alerts [8]. All the protocols used in MANET can also be used in VANET [9]. Various research works have been taken so far in vehicular network but no work has been taken to establish GSM based wireless communication in vehicular network.

When we go through the previous works carried out so far indicates clearly that all the works concentrated establishing communication among vehicle and Road Side Unit (RSU) [1] [2]. The vehicle move on the road could be able to communicate with Road Side Unit [3] [4]. This road side unit is established with the help of towers, which are highly expensive. The vehicle move on the road is always connected with the road side unit in the form of wireless medium to pass the message. The road side unit receives the message from one vehicle and forward the message to the other vehicles that move on the road [3] [4]. Since the communication with the road side unit made up of towers are highly expensive, this form of communication cannot be established in all the countries. However the inter vehicle communication is highly required to communicate with the others vehicles while moving.

So a new way of communication model is need, which will be less cost effective. Another new approach was developed by forming network communication among vehicles by Bluetooth technologies [1] [2]. The inter vehicle wireless communication established by Bluetooth technology helps a lot to the vehicular network to communicate with one another. This network communication allows vehicles to send message to another vehicle, while moving on the highway. This communication module helps a lot during emergency cases. The network communication established by Bluetooth technology should be prevented from the hackers [2]. Fair architecture developed to prevent the network communication established by Bluetooth from the hackers. The communication established with the help of Bluetooth technology will lead to out of coverage, while the vehicle communicate with one another. The communication range of Bluetooth is just 10 meter, which will lead to out of coverage. Hence the communication by Bluetooth technology cannot establish a fair communication among the vehicles that move on the road. The communication with the help of road side unit is very costly, which cannot be formed in all the countries. The communication with Bluetooth technology will lead to out of coverage area often, since the vehicle move faster on the highway.

However a new communication model id needed to overcome the disadvantages of the existing communication model. The proposed GSM communication model will overcome the disadvantages of the existing communication model and this GSM communication model will bring out reliable communication among vehicles, mobile phones, help lines and home based telephones. The GSM technology is already available in all the countries, so forming network communication with the help of GSM technology is quite easy and less cost effective. Once GSM communication is implemented in the vehicles, the entire vehicle history can be tracked regardless wherever the vehicle has moved. In this paper, we have modeled GSM based network communication in VANET.

\section{STEM MODEL}

\section{A. Architecture Representation}

The Fig. 2 describes he entire architecture model of the proposed system. As indicated in the architecture, the GSM based mobile network vehicle can establish the 
communication with the other GSM enabled vehicle, Mobile phones, Help lines and home based telephone. The proposed GSM based mobile network communication will bring out certain useful metrics, as shown in the architecture diagram. Establishing the communication model among vehicle, mobile phone, help line and home based telephone using GSM based mobile network communication lead to the following metrics, such as SMS, MMS, Voice call, Help line and Vehicle tracking model.

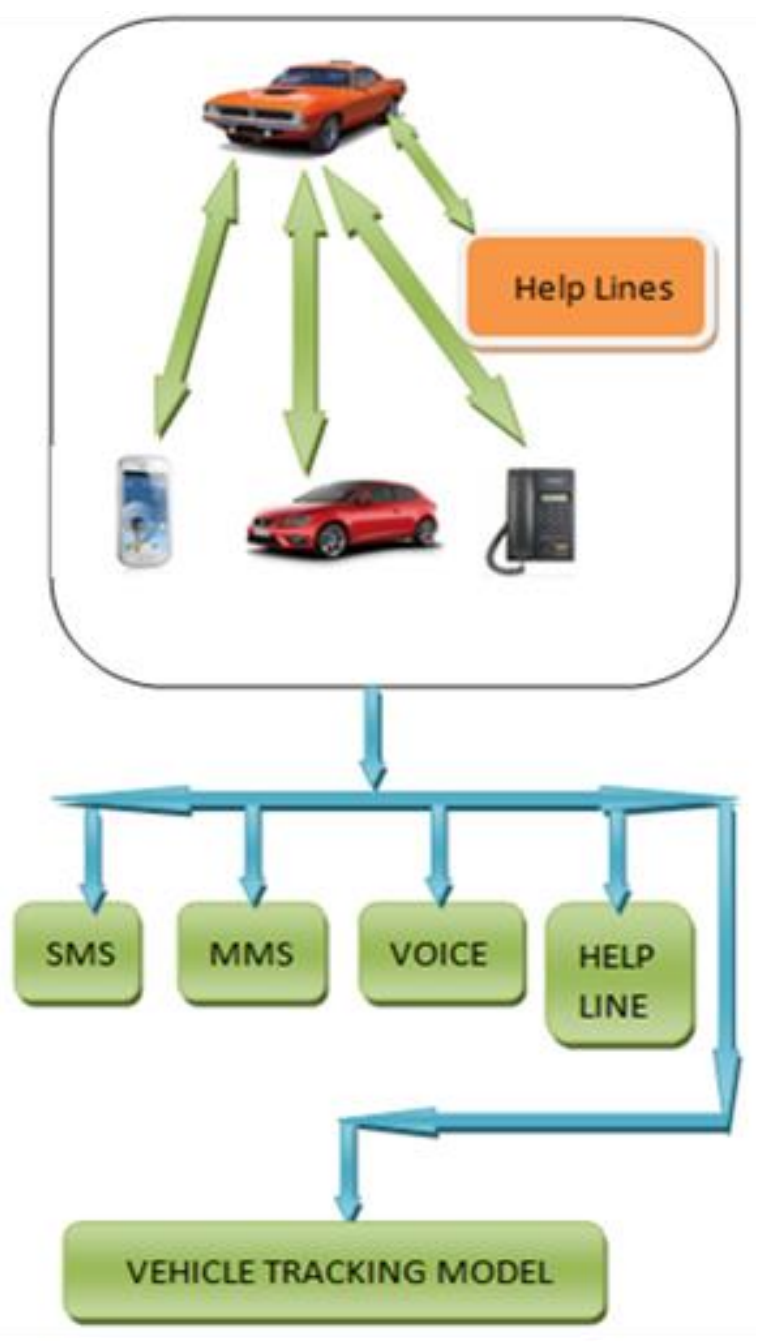

Figure 2: Architecture Representation

\section{B. GSM Enabled Vehicle}

The proposed system model is based on GSM network technology. Every vehicle is manufactured at the manufacturing factories and designed in a luxuries way. However we propose, all the vehicles should be manufactured with GSM based network model to take effect of the proposed system. The GSM based network device must be placed at the middle of the front of the vehicle and that could be able to operate by the both driver and the co-passengers, whoever sits in the front seat. The device should come out with the touch pad to operate the GSM network device on the vehicle and it should come out with the head phone to answer the voice call and so on. As we all know that every mobile phone should get charged to continue functioning. However the GSM based network mobile device installed at the vehicle should be designed in such a way that gets charging, while the vehicle is moving on the highway and the backup should remain, when the vehicle is not functioning. Once the device is installed properly, the SIM card can be inserted into the vehicle for establishing the communication. Getting the signal (tower) strength could be achieved by the same signal towers used to get the signals for the hand based mobile phones. This type of manufacturing vehicle will establish the new way of network communication among vehicles and with other communicating devices such as mobile phones, help lines and home based telephones ultimately. If the proposed system come to effect, it will be helpful almost all the passengers for they can communicate with other sources and the vehicle can be easily tracked for its entire history. For instance, if a vehicle is stolen or hijacked it could be easily tracked wherever the vehicle has moved by the GSM based network communication established in the vehicle.

\section{Performance Evaluation}

This type of installing GSM network model device in the vehicle will bring out all the benefits available in the normal mobile phones, because the same mobile phone device is manufactured with the vehicle to form the network communication. Some of the major benefits of the proposed system is listed and described below.

- $\quad$ Short Message Service (SMS)

- Multimedia Message Service (MMS)

- Voice Call

- Help Line Communication

- Vehicle Tracking Model

\section{a. Short Message Service (SMS)}

Short Message Service (SMS) is a process of sending short message to other devices through a mobile network. Vv cv cvc cv vc cv cv vc When GSM based mobile network is installed in the vehicle, the vehicle could be able to send short messages to other devices at any time. This message services will be helpful to the passengers to communicate with other sources such as mobile phones, help lines, home based telephones and even to other GSM based vehicles. When a vehicle move on the highway, this messaging service will be helpful a lot to send short messages to the others sources and even to the help lines found on the highway. To make the scheme work effectively the following proposed methodology should be maintained. A separate SIM card should be sold at the shop that is only designed for the vehicles. This SIM card must contains all the help line numbers sate wise stored in a predefined manner. The short message service should be allowed at free of cost to the help line numbers. Thus the short message service will 
bring out the safety of the passengers, while moving on the vehicle because they could be able to send short messages to the help lines and other sources during the emergency situations.

As represented in the Fig. 3 the short message service (SMS) can be sent to other vehicles, mobile phones and mainly to the help lines, which will be helpful to bring out the safety of the passengers.

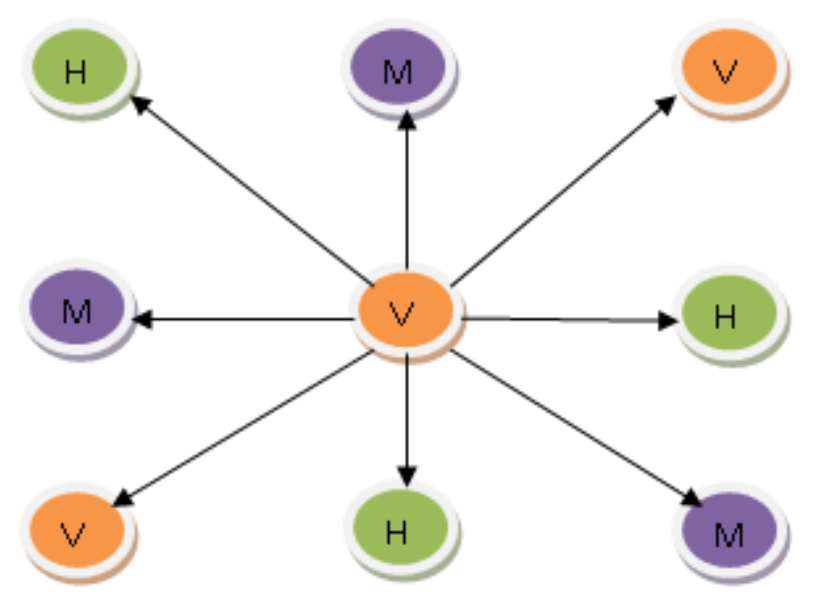

V- Vehicle, M- Mobile, H- Help Line

Figure. 3: SMS Communication Model

\section{b. Multimedia Messaging Service (MMS)}

Another benefit of modeling GSM based mobile network communication in vehicular network is passing Multimedia Messaging Service (MMS) formally known as MMS. This facility helps a lot to pass or send some sort of images or pictures and short video clips from one device to other devices. When GSM based mobile network is incorporated in the vehicle, the vehicle can be able to send MMS to the help lines and other sources such as mobile phones and so on. This feature allows the vehicles to send the road condition status and even any mishap happened on the highway may be communicated with the help lines (all the help lines numbers are stored already state wise) and necessary action can be taken as early as possible. However, due to rain or any weather conditions the road condition might be changed, which may lead to major accidents. This MMS feature will help a lot to prevent it by communicating with the help line in time.

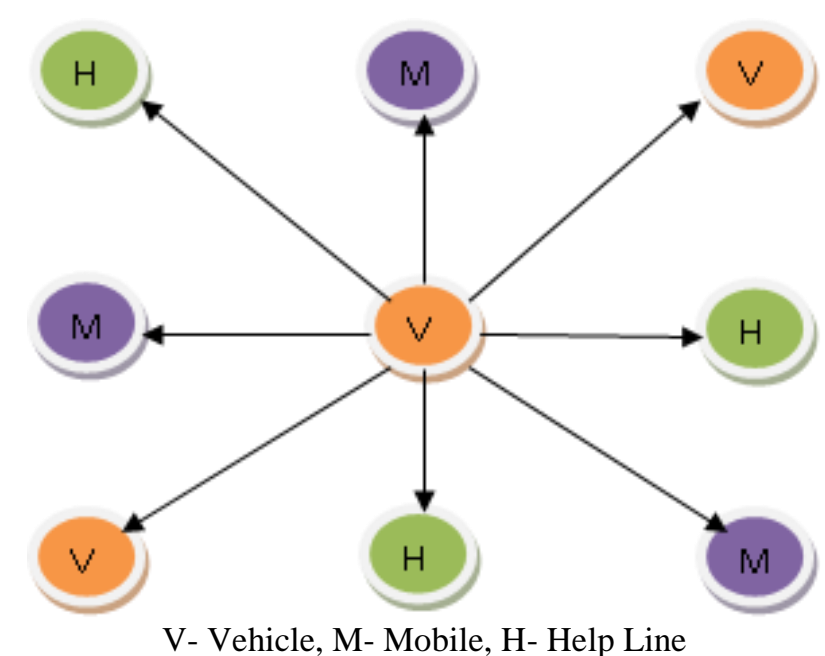

Figure. 4: MMS Communication Model

The Fig. 4 shows the way of passing MMS to the other help lines, mobile phones and other GSM based vehicles, which will bring to the notice of the road condition and some sort of mishap happened on the highway.

\section{c. Voice Call}

Voice call facility is the major added advantage of GSM based mobile network communication. Using this facility, the vehicle could communicate with any other vehicle, mobile phones and help lines at any time. The users can answer the voice call or make voice call with the help of headphone associated with it. This voice can be connected with any source at any time. However this metric will be helpful a lot to make calls to the help lines at the highway during emergency situations such as break down of the vehicle, no fuel and so on. All the help lines numbers should be stored at the SIM card state wise and the voice call should be connected at any time. The proposed system enables GSM based mobile network communication among the vehicle, mobile phones, help lines and home based telephones. The only thing is the signal strength should be perfect at the highway also. Thus, the proposed GSM based mobile network communication brings the effective communication in vehicular network and with other network sources.

\section{d. Help Line Communication}

At every highway, we could find help line units to help the vehicle during emergency situations. However these help lines is available at longer distance and they may not come to know, what happened at long distance and they may not be able to help the vehicle in time. In order to help the vehicle in time at emergency cases, it is obviously GSM based mobile network communication is needed. Once GSM based mobile network communication is established at every vehicle, the vehicle will have all the help line numbers stored state wise available in all over the country. This sort of help lines numbers stored already at the SIM card will help the vehicle to communicate with the 
help lines at any time to extend some sort of help from them. While we travel on the highway, we could find the road condition unsafe and that could be informed to the help lines to take the immediate action at once. However the vehicle may get break down on highway especially during night times, those times the vehicle must need the help from the help units found in the highway. In these situations communication with the help lines is must. So to make a reliable communication with the help line in time, the GSM based mobile network communication is needed. This communication will help a lot, when some sort of mishap found on the highway and these communication will prevent others mishaps in the highway. So network communication to the help lines is ultimately needed.

\section{e. Vehicle Tracking Model}

The major add metric of this GSM based mobile network communication is vehicle tracking Model. The benefit of this metric is the entire vehicle history and the current location of the vehicle can be easily identified. The total distance traveled by the vehicle and the total time taken to travel the distance can be identified by the GSM based mobile network communication in vehicular network. This added advantage will be helpful to identify the vehicle, when the vehicle is stolen or hijacked by the hijackers. Thus, the above major advantages proposed above prove the worth of the GSM based mobile network communication in vehicular network.

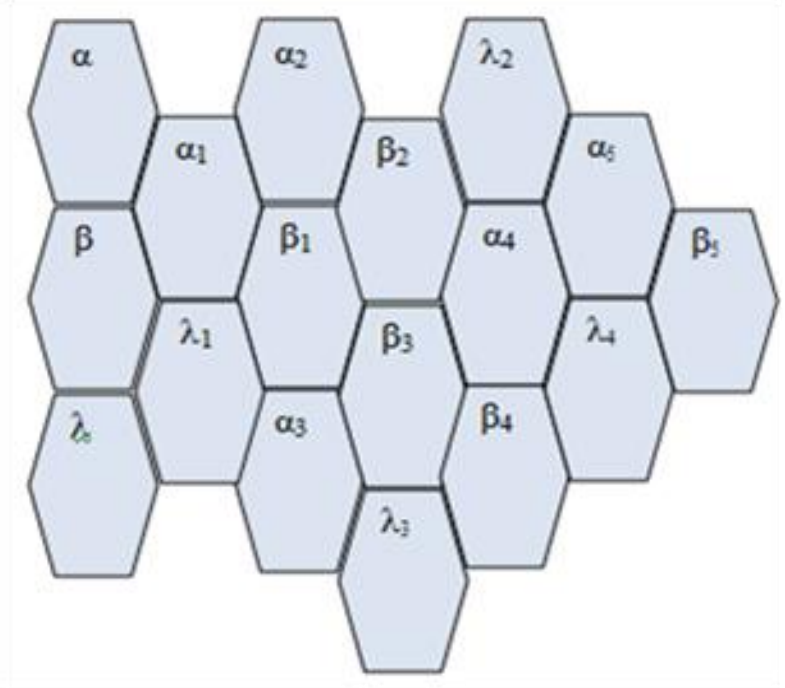

Figure. 5: Cell Representation in GSM Network

The above Fig. 5 shows the cell representation in GSM mobile network. Each cell is named with unique name for its identification. Each cell is active area, where mobile network signal (tower) is available. Every highway is covered with the active cell to produce the continuous signal strength for better communication. The vehicle tracking system could be achieved with the help of these active cells. When a strength from any one of the active cell with the help of this active cell, we can identify the current location of the vehicle and where the vehicle is currently moving towards further with the next active cell, when vehicle reaches. Thus, the location of the vehicle and the entire vehicle moving history can be easily identified. This information will be helpful a lot to identify the vehicle, when it has been stolen or hijacked.

The above Fig. 6 shows the vehicle route from source (S) to destination (D). The vehicle moves on the highway within the active cell region, so the vehicle route and its current location can be easily tracked.

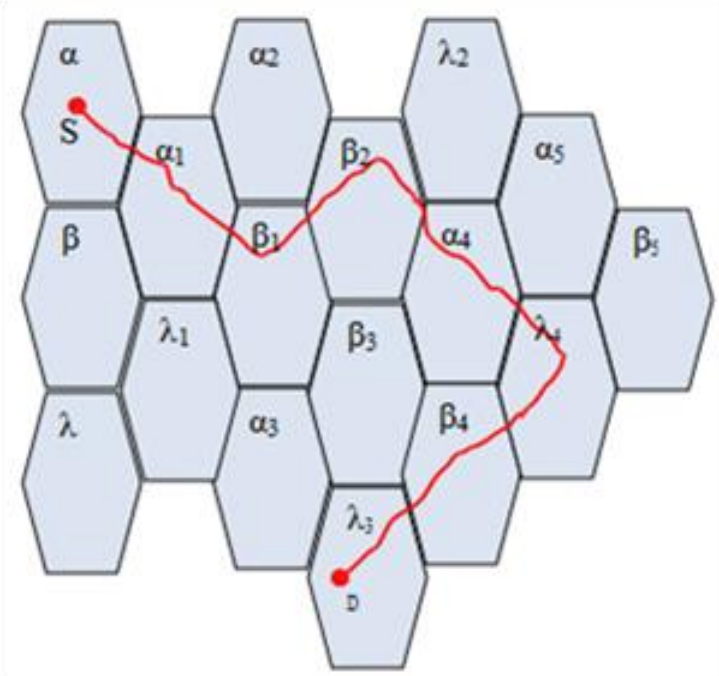

Figure. 6: Vehicle moving from source to destination within the active cells.

As indicated in the Fig 6, the vehicle moves from source (S) to destination (D) with the following active cells $\alpha, \alpha 1, \beta 1, \beta_{2}, \alpha_{4}, \lambda 4, \beta 4, \lambda_{4}$. Let's consider each active cell covers 50 meters distance and the vehicle moves $100 \mathrm{kmph}$. The current location of the vehicle is known by the active cell's unique name and distance traveled by the vehicle and the time taken to travel the distance can be calculated by the values as shown below. The total distance traveled by the vehicle can be calculated as follows.

$\mathrm{DT}=\alpha+\alpha_{1}+\beta_{1}+\beta_{2}+\alpha_{4}+\lambda_{4}+\beta_{4}+\lambda_{4}$

From the equation (1), DT refers the total distance traveled by the vehicle. Let's consider each active cell's coverage is $50 \mathrm{~m}$, so the total distance traveled by the vehicle could be identified easily.

$$
\begin{aligned}
& \mathrm{DT}=50+50+50+50+50+50+50+50 \\
& \mathrm{DT}=400 \mathrm{~m}
\end{aligned}
$$

Similarly the total time taken to reach the DT can be calculated as shown below. T $=\mathrm{DT} / \mathrm{SP}$

Where, 


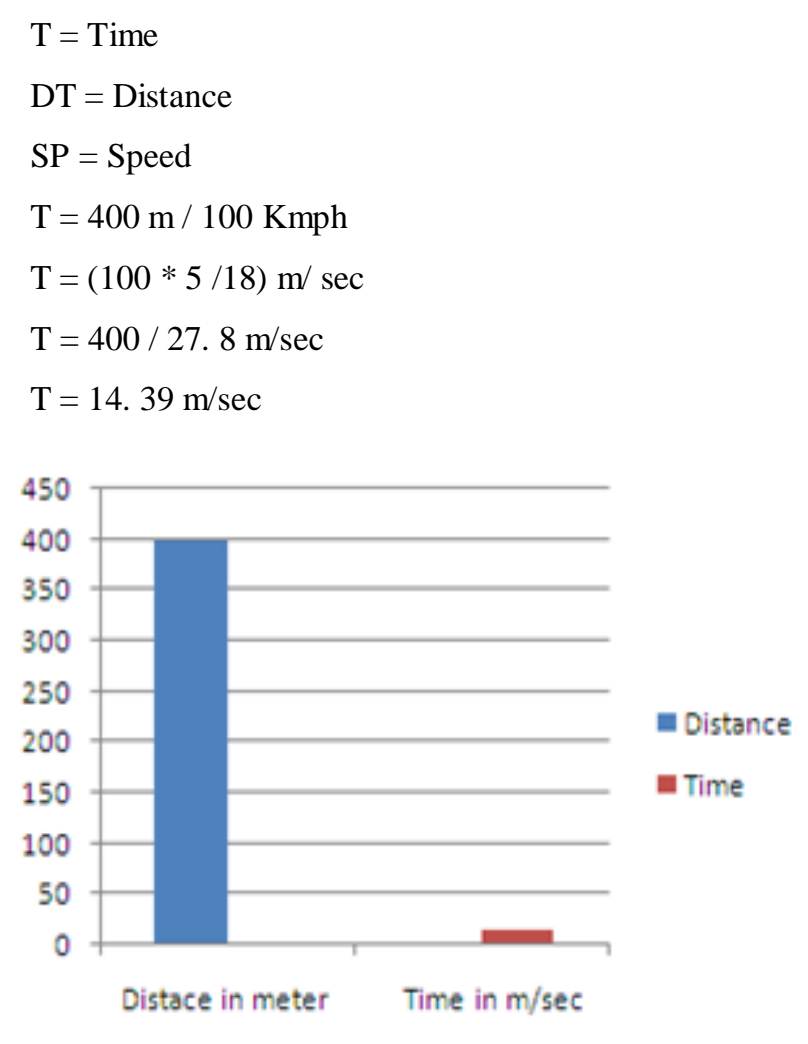

Figure. 7: Distance and Time

The Fig. 7 shows the distance traveled by the vehicle and the time taken to reach the distance based on the above calculation. The total distance traveled is $400 \mathrm{~m}$ and the time taken to reach the distance is $14.39 \mathrm{~m} / \mathrm{sec}$. As described above the total distance and total time taken can be calculated. Thus, the vehicle tracking model proves its worthiness.

\section{CONCLUSION}

In this paper, we have studied the comprehensive characteristic of vehicular network and we modeled a new GSM based mobile netw ork communication in vehicular network. The proposed GSM based network communication establishes network communication between vehicle, mobile phones, help lines and home based telephones. This GSM based network communication proves its effectiveness by the metrics obtained by the network communication. The metric of the GSM based mobile network communication are SMS, MMS, Voice call, Help line and Vehicle tracking Model.

\section{REFERENCES}

[1] M. Milton Joe, R.S. Shaji, R. Thulasi, "Modeling Network Communication in VANET using Bluetooth Technology", International Journal of Advanced and Innovative Research, Vol 2 No 3, PP. 643-651, March - 2013.
[2] M. Milton Joe, R.S. Shaji, K. Ashok Kumar,"Establishing Inter Vehicle Wireless Communication in Vanet and Preventing It from Hackers ", IJCNIS, vol.5, no.8, pp.55-61, 2013. DOI: 10.5815/ijcnis.2013.08.07.

[3] B. Ramakrishnan, Dr. R. S. Rajesh, R. S. Shaji, "An efficient vehicular communication outside the city environments" International Journal of NextGeneration Networks (IJNGN) Vol.2, No.4, December 2010.

[4] B.Ramakrishnan, Dr.R.S.Rajesh, R.S.Shaji, “An Intelligent Routing Protocol for Vehicle safety communication in Highway Environments", journal of computing-Volume 2, Issue 11, November 2010dec 2010.

[5] Saxena, N.; Tsudik, G.; Jeong Hyun Yi; Polytech. Univ., Brooklyn'Efficient Node Admission and Certificateless Secure Communication in Short-Lived MANETs", Parallel and Distributed Systems, IEEE Transactions - Feb. 2009, Volume: 20 Issue: 2 on page(s): $158-170$.

[6] Lin Yang Jingdong Xu Gongyi Wu Jinhua Guo, Nankai Univ Tianjin, China "Road Probing: RSU Assisted Data Collection in Vehicular Networks" Wireless Communications, Networking and Mobile Computing, Beijing, page: 1-4.

[7] Wern-Yarng Shieh Wei-Hsun Lee Shen, "Analysis of the Optimum Conuration of Roadside Units and

On board Units in Dedicated Short-Range Communication Systems", Intelligent Transportation Systems, IEEE Transactions on Issue Date : Dec. 2006 Volume :7, Issue:4 On page(s): 565 - 571.

[8] Biswas, S. Misic, J. "Proxy signature-based RSU message broadcasting in VANETs", Communications (QBSC), 2010 25th Biennial Symposium on Date: 12-14 May 2010 on page(s): 5 - 9 Location: Kingston.

[9] Tuteja, AsmaGujral, Rajneesh Thalia, Sunil," Comparative Performance Analysis of DSDV, AODV and DSR Routing Protocols in MANET Using NS2", ACE, International Conference on 2010, page(s): $330-333$.

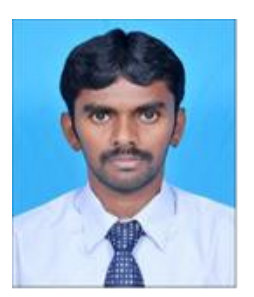

Mr. M. Milton Joe received his B.Sc Computer Science degree from Bharathidasan University,India and MCA degree from Anna University in India. Presently he is working as Assistant Professor at St. Jerome's College in Nagercoil, India. He has two years of research experience and authored seven research papers in reputed international journals. His research interests include Network Security, Network Communication, Vehicular Network and Social Networks. 


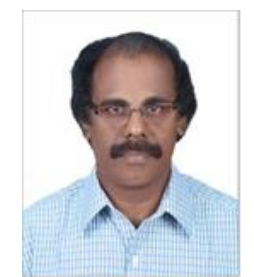

Dr. B. Ramakrishnan is currently working as Associate Professor in the Department of Computer Science and research Centre in S.T. Hindu College, Nagercoil. He received his M.Sc degree from Madurai Kamaraj University, Madurai and received Mphil (Comp. Sc.) from Alagappa University Karikudi. He earned his Doctorate degree in the field of Computer Science from Manonmaniam Sundaranar University, Tirunelveli. He has a teaching experience of 26 years. His research interests lie in the field of Vehicular networks, mobile network and communication, Cloud computing, Green computing, Ad-hoc networks and
Network security.

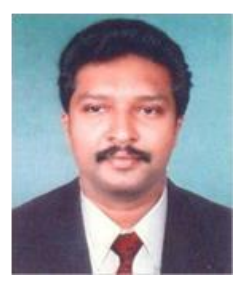

Dr. R.S. Shaji received his M.Tech in Computer Science and Engineering from Pondicherry University. $\mathrm{He}$ earned his Doctorate degree in the field of Computer science and Engineering from Manonmaniam Sundaranar University. Presently he is working as Professor in Noorul Islam University. He has eight years of research experience and published more than twenty five international journals. His research interests include Mobile and pervasive Networks.

How to cite this paper: M. Milton Joe, B. Ramakrishnan, R. S. Shaji,"Modeling GSM Based Network Communication in Vehicular Network", IJCNIS, vol.6, no.3, pp.37-43, 2014. DOI: 10.5815/ijcnis.2014.03.05 